\title{
Evaluation of Saccharomyces pastorianus impact to Sauvignon blanc chemical \& sensory profile compared to different strains of S. cerevisiae/bayanus
}

\author{
V. Troianou ${ }^{1}$, C. Toumpeki ${ }^{1}$, E. Dorignac ${ }^{2}$, C. Kogkou ${ }^{3}$, S. Kallithraka ${ }^{3}$, and Y. Kotseridis ${ }^{3}$ \\ ${ }^{1}$ Innovino, Applied Research for Wine, Pallini, Athens \\ ${ }^{2}$ Société Industrielle Lesaffre, Division Fermentis, France \\ ${ }^{3}$ Laboratory of Enology, Department of Food Science \& Human Nutrition, Agricultural University, Athens
}

\begin{abstract}
Enhancing flavors and/or improving fermentation parameters through the use of different species of yeast strains is nowadays a frequent challenge in winemaking research, especially for aromatic varieties such as Sauvignon Blanc. In this work, the aim was to focus on the impact of a species not already studied in wine: Saccharomyces pastorianus. Twenty-two fermentations were conducted on a Sauvignon Blanc must by addition of different strains and mixtures of them by using two different inoculation temperatures. The must was inoculated in cold condition with two similar mixtures of $S$. pastorianus 1 or $2(70 \%)$ and $S$. bayanus (30\%), with S. pastorianus 1 or 2 alone, with the correspondent $S$. bayanus alone and with two other $S$. cerevisiae 1 and 2 alone as well. For classic condition, the must was inoculated with only one mixture $S$. pastorianus $1(70 \%)$ / S. bayanus (30\%), and respectively with S. pastorianus 1 , S. bayanus and S. cerevisiae 2 alone. Samples were taken all along the fermentations for both conditions in order to check chemical and microbial analyses as well as yeast implantations. The final wines were analysed for alcohol, glucose, fructose, all other classical wine analysis as well as for acetate esters, and higher alcohols. The results underlined that for both $S$. pastorianus 1 and $S$. pastorianus 2 strains, the production of acetic acid was zero in cold condition and really low $(0.09 \mathrm{~g} / \mathrm{l})$ for classic condition regarding $S$. pastorianus 1 . As a consequence, Saccharomyces pastorianus seems to be highly interesting for winemaking, alone or in co-inoculation with S. bayanus.
\end{abstract}

\section{Introduction}

Lager beer is produced industrially using Saccharomyces pastorianus, a natural hybrid of Saccharomyces cerevisiae and Saccharomyces eubayanus [Nakao et al., 2009; Libkind et al., 2011]. The name was delivered in honor of Louis Pasteur. Saccharomyces pastorianus is a bottom fermenting yeast, resistant to low temperatures, which flocculates well, sediments fast and ferments glucose, fructose but also complex sugars as maltose and maltotriose in beer worts. Nowadays there is an increasing interest on yeast as yeast is considered to be the flavor engine of the wine and brewing industry [Ribereau Gayon, 2006, Meier-Dörnberg, 2017]. Yeast metabolism during the fermentation and ripening process gives rise to approximately $80 \%$ of all aroma-active compounds in beer, thereby determining its aroma profile [MeierDörnberg, 2017]. Among wine and beer yeasts, only some Saccharomyces cerevisiae strains are considered to be able to ferment satisfactory at low temperatures. Cryotolerant strains ferment well on temperatures from 6 to $30^{\circ} \mathrm{C}$ with an optimum around $25-30^{\circ} \mathrm{C}$. Walsh \& Martin (1977) reported that these cryotolerant S. cerevisiae strains belong more frequently to the p.r. uvarum (strains fermenting melibiose) but also to p.r. bayanus (strains not fermenting galactose) [Castellari et al. 1994, Massoutier et al.,1998]. Cryotolerant strains possess a number of advantages compared with non-cryotolerant strains as less acetic acid and ethanol producers (Castellari et al.,
1994). Saccharomyces bayanus var. uvarum strains were also described by different authors as producing high concentration of volatile fermentative compounds such as phenylethanol and its acetate. In this work, the aim was to focus on the impact of the species Saccharomyces pastorianus not yet studied in wine. It is a species close to Saccharomyces uvarum and Saccharomyces bayanus however different.

\section{Materials and methods}

\subsection{Must preparation and conditions}

$100 \mathrm{~kg}$ of white grapes (variety Sauvignon Blanc) were used during this experimentation. After grape crushing, the must was sulfited with the addition of $80 \mathrm{mg} / \mathrm{l}$ metabisulfite and then subjected to clarification by cold/gravity settling and then decanted. Almost $60 \mathrm{~L}$ of clarified must were prepared and split in 22 recipients. 22 experimental fermentations (11 duplicated trials) were conducted by addition of different strains (and mixtures of them) and by using two different inoculation temperatures. In cold condition, yeasts were inoculated when the temperature reached $13{ }^{\circ} \mathrm{C}$ and in classic condition yeasts were inoculated when temperature reached $17^{\circ} \mathrm{C}$. Inoculation rate was $200 \mathrm{mg} / \mathrm{l}$.

The strains and the corresponding codes are shown in Table 1. 
Table 1. Conditions, codes and yeast strains.

\begin{tabular}{lll}
\hline CONDITION CODES & YEASTS STRAINS \\
\hline COLD & & \\
\hline 1 & Sb-P1 & $\begin{array}{l}\text { Mix S. pastor } 1.70 \% \text { \& S. bayanus } \\
30 \%\end{array}$ \\
2 & Sb-P2 & $\begin{array}{l}\text { Mix S. pastor } 270 \% \text { \& S. cerevi } 1 \\
30 \%\end{array}$ \\
& & S. pastorianus 1 \\
4 & P1 & S. pastorianus 2 \\
5 & P2 & S. bayanus \\
6 & Sb & S. cerevisiae 1 \\
7 & Sc1 & S. cerevisiae 2 \\
\hline ClASSIC & Sc2 & \\
\hline 8 & Cl Sb-P1 & Mix S. pastor $170 \%$ \& S. bayanus \\
& & $30 \%$ \\
9 & Cl P1 & S. pastorianus 1 \\
10 & Cl Sb & S. bayanus \\
11 & Cl Sc2 & S. cerevisiae 2 \\
\hline
\end{tabular}

\subsection{Chemical and microbiological analyses}

The must was analyzed immediately for the following parameters: glucose/fructose, total acidity, $\mathrm{pH}$, malic acid, yeast assimilable nitrogen (YAN), free and total $\mathrm{SO}_{2}$ and turbidity using OIV methods. The analysis of the initial must and the applied method for each parameter are shown in Table 2.

After the completion of fermentations (PCR analyses confirmed that all the inoculated yeasts were implanted very well and achieved the completion of alcoholic fermentation, proving that not any cross-contamination happened during the experimentation), the following parameters were measured and evaluated: total acidity (TA), pH, malic acid (AM), lactic acid (LA), volatile acidity (VA in $\mathrm{g} / \mathrm{L}$ of acetic acid) and also glycerol $(\mathrm{g} / \mathrm{L})$. In Table 3, alcohol levels and fructose to glucose ratio are shown. Volatile compounds were measured using Gas Chromatography Mass Spectrometry and the levels of 2- phenyl ethanol and its acetate are shown in Fig. 5.

\section{Results and discussion}

\subsection{Must analysis}

The Sauvignon blanc must was relatively rich in sugar content and malic acid. Since the initial YAN was $169 \mathrm{mg} / \mathrm{L}$, the must have been supplemented with the following nutrients: $24 \mathrm{hrs}$ after the yeast addition: $200 \mathrm{mg} / \mathrm{l}$ SpringFerm ${ }^{\mathrm{TM}}$ (yeast autolysate) Fermentis. After one third of fermentation completion: $240 \mathrm{mg} / \mathrm{L}$ of diammonium phosphate (DAP) $+200 \mathrm{mg} / \mathrm{L}$ SpringFerm ${ }^{\mathrm{TM}}$ - Fermentis. According to these results the must presents a probable alcoholic degree (PAD) of $12.76 \% \mathrm{v} / \mathrm{v}$.

\subsection{Wine analyses}

The fermentations were completed after 9 days of fermentation for the classic $\mathrm{Sb}$, at 12 days for cold and classic Sc2, at 18 days for cold and classic P1 and P2, at 11 days for all the other samples Sb-P1, Sb-P2, Sc1, cold $\mathrm{Sc} 1$, cold $\mathrm{Sb}$ ). No significant differences were recorded for alcohol level and the same was found for the sugars to alcohol yield with a lowest value of 16.8 to a highest of
Table 2. Analysis of the must just after clarification.

\begin{tabular}{ll}
\hline PARAMETER & RESULT \\
\hline Glucose $(\mathrm{g} / \mathrm{L})$ & 114 \\
Fructose $(\mathrm{g} / \mathrm{L})$ & 103 \\
Glucose + Fructose & 217 \\
(g/L) & \\
Total Acidity $(\mathrm{g} / \mathrm{L}$ & 5.1 \\
tartaric acid) & \\
Malic acid $(\mathrm{g} / \mathrm{L})$ & 3.84 \\
YAN (primary amino & 142 \\
nitrogen) (g/L) & \\
YAN $(\operatorname{ammonia})(\mathrm{g} / \mathrm{L})$ & 27 \\
Total $\mathrm{YAN}(\mathrm{g} / \mathrm{L})$ & 169 \\
Free $\mathrm{SO}_{2}(\mathrm{mg} / \mathrm{L})$ & 17.1 \\
Total $\mathrm{SO}_{2}(\mathrm{mg} / \mathrm{L})$ & 30 \\
\hline
\end{tabular}

Table 3. Alcohol levels of the samples.

\begin{tabular}{llll}
\hline Samples & Fr/Gl ratio & \%Vol & g sugars/Alc \\
\hline S103-P1 & 0.02 & 12.75 & 17.0 \\
S103-P2 & 0.03 & 12.7 & 17.1 \\
P1 & 1.72 & 12.8 & 16.8 \\
P2 & 0.75 & 12.85 & 16.8 \\
S103 & 0.07 & 12.65 & 17.1 \\
S102 & 0.90 & 12.85 & 16.8 \\
S104 & 0.89 & 12.6 & 17.2 \\
Cl S103-P1 & 0.04 & 12.85 & 16.9 \\
Cl P1 & 0.23 & 12.65 & 17.1 \\
Cl S103 & 0.05 & 12.8 & 16.9 \\
Cl S104 & 1.86 & 12.75 & 16.9 \\
\hline
\end{tabular}

17.2. The fructose to glucose ratio was relatively elevated for P1 and P2, however it was high for the Saccharomyces cerevisiae samples also. Noteworthy was the ratio found for the $S$. bayanus samples for which the values recorded were around zero meaning that $S$. bayanus assimilated equally both fructose and glucose. S. bayanus is adequate for fructose degradation which is the case of maturity grapes, being rich in fructose and sometimes presenting higher levels of fructose than glucose.

These findings indicate that Saccharomyces pastorianus achieved the completion of fermentation the same as happened for Saccharomyces cerevisiae and Saccharomyces bayanus despite the longer fermentation period for achieving that.

\subsection{Volatile acidity}

At the end of the fermentation the samples were analysed for acetic acid production (Fig. 1). Noteworthy was the non-production of acetic acid by both Saccharomyces pastorianus strains. Combination of $S$. bayanus and $S$. pastorianus resulted to higher acetic acid production however lower than that produced for Sc2 and $\mathrm{Cl} \mathrm{Sc2}$. The acetic acid levels were well below the maximum permitted limit. This result is of a high interest for winemakers especially in warm climate regions where over-ripeness is most of the time the case and they should find ways to avoid high volatile acidities. 


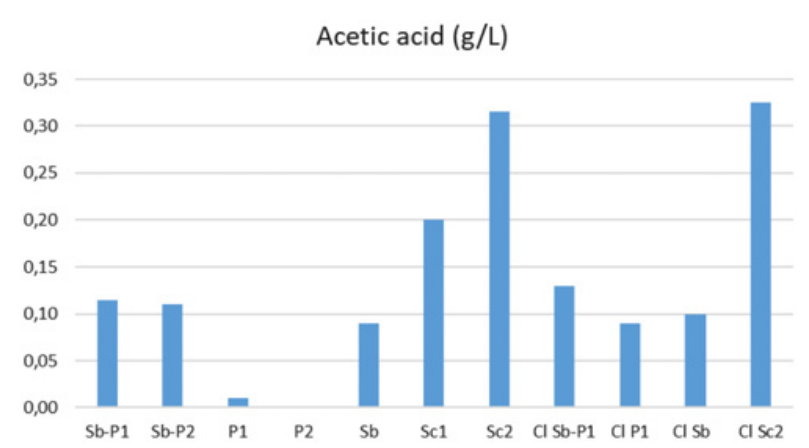

Figure 1. Levels of acetic acid production. S. pastorianus resulted to zero acetic acid production.

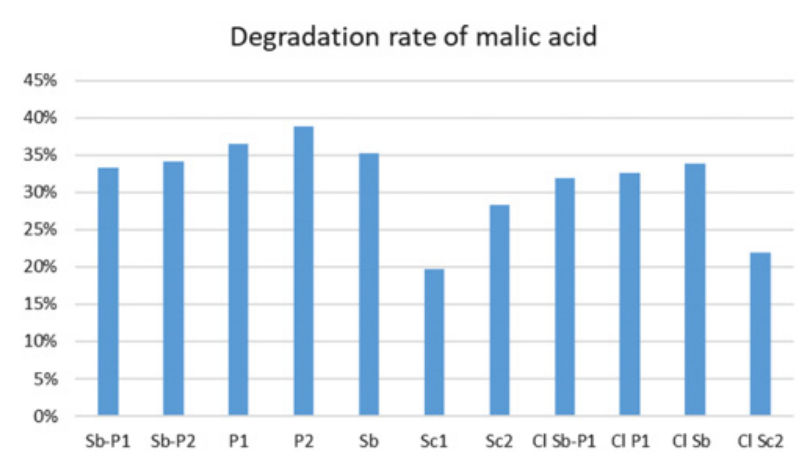

Figure 2. Degradation rate of malic acid.

\subsection{Malic acid conversion}

S. pastorianus resulted to higher $\mathrm{pH}$ values and lower titratable acidity levels (data not shown). These findings are partially explained by the higher degradation level of malic acid achieved by $S$. pastorianus yeasts. Considering the malic acid degradation, it seems that S. pastorianus degrades higher amounts of malic acid than other yeasts. Cold condition seemed to increase the level of degradation (Fig. 2). Considering lactic acid results, no malolactic fermentation occurred, none of these strains produced lactic acid. Malic acid was rather transformed to ethanol through a malo-ethanolic conversion.

Apparently, S. pastorianus is more efficient on malic acid transformation and this could be beneficial for cold climate winemaking were the levels of malic acid are high. $\mathrm{Sb}$ also achieved high degradation levels of malic acid, while $\mathrm{Sc} 1$ and $\mathrm{Sc} 2$ recorded less malic acid degradation.

\subsection{Glycerol production}

Glycerol production was evaluated for all the yeasts. Glycerol is considered to contribute to body sensation as also to aftertaste. Wine makers and wine writers consider that glycerol, ethanol and sugar are the principal components of the overall mouthfeel of white or red wines. Few researches refer to glycerol (Nurgel \& Pickering, 2005; Pickering et al. 1998). According to Yanniotis, et al. (2007) a multiple linear model describing first order effects of ethanol, dry extract and glycerol on the viscosity and thus on wine mouthfeel of the wine was plotted by the results found for white wines.

Considering glycerol production, the pure S. pastorianus samples achieved higher amounts of glycerol. Classic condition seemed to increase glycerol production in

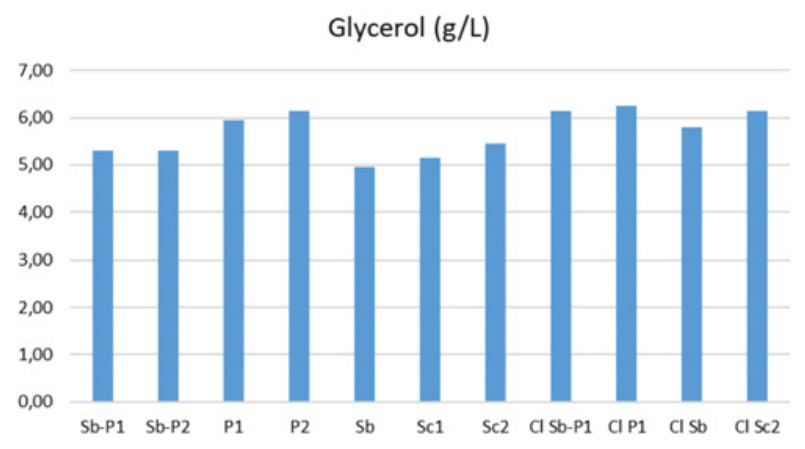

Figure 3. Levels of glycerol produced by the different yeasts.

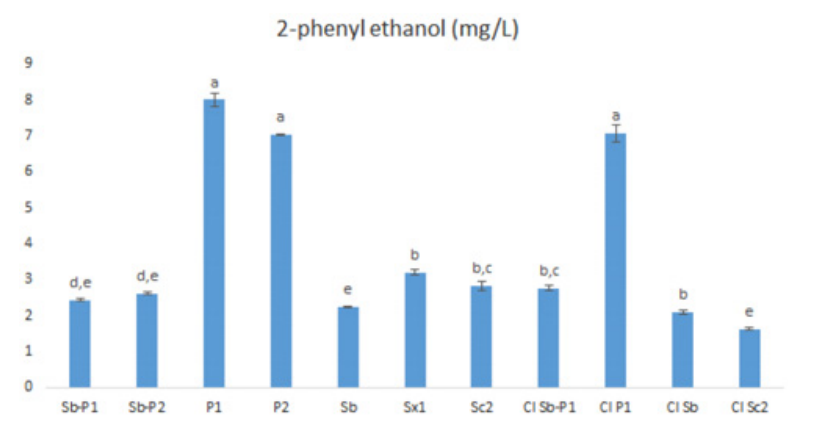

Figure 4. Levels of 2-phenyl ethanol $(\mathrm{mg} / \mathrm{L})$ by the different yeasts.

comparison to cold inoculation conditions. The S. bayanus and $S$. cerevisiae 1 and 2 achieved lower levels of glycerol at our experimentation indicating that $S$. pastorianus could induce higher wine mouthfeel.

\subsection{Volatile compounds production}

As known, yeast strain modulates the level of volatile compounds produced during alcoholic fermentation which monitors wine aroma. Ethylic esters as also acetates contribute decisively to wine aroma. Among these compounds 2-phenylethanol and its acetate contribute positively to wine aroma with rose, honey-like aromas and considered to be as beneficial for white wine aroma. $S$. bayanus var. uvarum strains were also described by different authors as producing high concentration of volatile fermentative compounds such as 2-phenylethanol and its acetate. These compounds were measured during this study and the ability of each strain was evaluated towards the levels of these beneficial for wine aroma compounds (Fig. 4).

According to the results found Saccharomyces pastorianus 1 achieved significantly higher levels of 2phenyl-ethanol in both conditions, cold inoculation (P1) as also in classical conditions ( $\mathrm{Cl} \mathrm{P} 1)$. It was noteworthy that Saccharomyces pastorianus 2 did produce as well high levels of this rose aroma compound in the cold inoculation conditions, while $\mathrm{Cl} \mathrm{Sb}$, the sample of $\mathrm{S}$. bayanus inoculated at classical conditions achieved levels as low as that found for $S$. cerevisiae 1 and 2 .

As expected similar trends were found for the acetate of 2-phenyl ethanol, which could be considered as more powerfull aromatically presenting lower perception threshold than its corresponding higher alcohol (Fig. 5). 


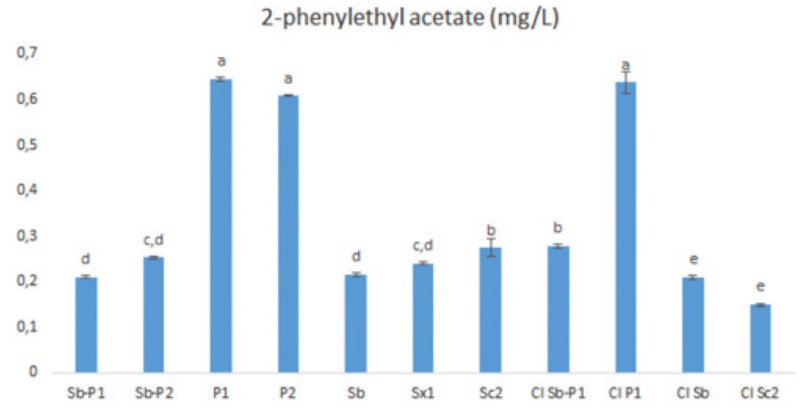

Figure 5. Levels of the acetate of 2-phenylethanol $(\mathrm{mg} / \mathrm{L})$ by the different yeasts.

\section{Conclusions}

Saccharomyces pastorianus was studied in grape juice fermentation for the first time using an aromatic variety such as Sauvignon Blanc. Relatively more days were necessary in order to achieve alcoholic fermentation completion in comparison to $S$. cerevisiae, $S$. bayanus and mixtures of $S$. bayanus and $S$. pastorianus. Despite this delay the alcohol level produced was same to the other species. Acetic acid production was almost zero, which makes these species highly interesting for quality winemaking. Someone could use these species in order to avoid high volatile acidity especially when the grapes are rich in sugars. Malic acid degradation is also a strong point for making this yeast of interest. The conversion of malic acid was up to $40 \%$. Besides, glycerol produced by this yeast was around $1 \mathrm{~g} / \mathrm{L}$ higher than $S$. bayanus and $S$. cerevisiae and this could result to wines with higher mouthfeel. Also S. pastorianus seemed to produce higher levels of rose like aromas in the wine.
Overall S. pastorianus could be an interesting alternative for wine making and thus further research should be devoted to these species.

\section{References}

[1] Y. Nakao, T. Kanamori, T. Itoh, Y. Kodama, S. Rainieri, N. Nakamura, et al., DNA Res. 16, 115 (2009)

[2] D. Libkind, C.T. Hittinger, E. Valerio, C. Goncalves, J. Dover, M. Johnston, et al., Proc. Natl. Acad. Sci. U.S.A. 108, 14539 (2011)

[3] Ribereau Gayon Handbook of enology. Volume 1, The Microbiology of Wine and Vinifications (Ed John Wiley 2006)

[4] T. Meier-Dörnberg, Genetic and phenotypic characterization of different top-fermenting Saccharomyces cerevisiae ale yeast isolates. In Proceedings of the 36th European Brewery Convention, Ljubljana, Slovenia, 14-18 May (2017)

[5] R.M. Walsh, P.A. Martin, J . Inst. Brew. 83, 169 (1977)

[6] L. Castellari, M. Ferruzi, A. Magrini, P. Giudici, et al., Vitis 33, 49 (1994)

[7] C. Massoutier, H. Alexandre, M. Feuillat, C. Charpentier, Vitis 37, 55 (1998)

[8] C. Nurgel, G. Pickering, J. Texture Studies 36, 303 (2005)

G. Pickering, D. Heatherbell, D. Barnes, L.P. Vanhanen, Amer. J. Enology and Viticulture 49, 306 (1998)

[9] S. Yanniotis, G. Kotseridis, A. Orfanidou, A. Petraki, J. Food Engin. 81, 399 (2007) 